\title{
Reversible microscale assembly of nanoparticles driven by the phase transition of a thermotropic liquid crystal
}

\author{
Niamh Mac Fhionnlaoich ${ }^{1}$, Stephen Schrettl ${ }^{2 \dagger}$, Nicholas B. Tito ${ }^{3}$, Ye Yang $^{1,2}$, Malavika Nair ${ }^{2 \ddagger}$, \\ Luis A. Serrano ${ }^{1}$, Kellen Harkness ${ }^{2}$, Paulo Jacob Silva ${ }^{2}$, Holger Frauenrath ${ }^{2}$, W. Craig Carter ${ }^{4}$, \\ Francesco Stellacci ${ }^{2}$, Stefan Guldin ${ }^{1,2 \star}$
}

The arrangement of nanoscale building blocks into patterns with microscale periodicity is challenging to achieve via self-assembly processes. Here, we report on the phase transition-driven collective assembly of gold nanoparticles in a thermotropic liquid crystal. A temperature-induced transition from the isotropic to the nematic phase leads to the assembly of individual nanometre-sized particles into arrays of micrometre-sized aggregates, whose size and characteristic spacing can be tuned by varying the cooling rate. This fully reversible process offers hierarchical control over structural order on the molecular, nanoscopic, and microscopic level and is an interesting model system for the programmable patterning of nanocomposites with access to micrometre-sized periodicities.

Apart from their widespread use in display technologies, liquid crystals (LCs) have attracted considerable interest in various fields of soft matter research. ${ }^{1-3}$ LCs offer intriguing opportunities for colloid science. ${ }^{4-6}$ The elastic distortion of the mesogen director field around colloidal inclusions, i.e., topological defects, enables colloidal assembly into organized patterns by long-range interactions. ${ }^{7}$ Examples include nematic dipoles that can lead to the formation of colloidal chains ${ }^{8,9}$ and nematic quadrupoles that enable the growth of $2 \mathrm{D}$ colloidal crystals. $^{9}$ Furthermore, the mesogen director field may be tailored through the particles' shape. ${ }^{10}$ The interplay of anisotropic elastic and weakly screened electrostatic interactions opens up pathways to combined orientational and positional order. ${ }^{11,12}$ Colloid-induced topological defects can act as templates for self-assembly processes on the molecular scale. ${ }^{13}$ Such LC-mediated colloidal interactions are size-dependent. ${ }^{14-16}$ Accordingly, the elastic interaction with the director field should vanish when the diameter of the colloidal particles is below the surface extrapolation length, $\lambda=K / W$, where $K$ is the elastic constant of the nematic LC and $W$ is the surface anchoring strength, a measure of the interaction between the particle surface and the mesogen. ${ }^{16}$ Depending on the type of mesogens and the nature of the colloidal surface, this typically results in a minimum size of around $10-100 \mathrm{~nm}$ below which no elastic distortion of the director field is expected.

Composites of LCs with nanoparticles (NPs) that are below this length scale are nevertheless promising candidates for novel optoelectronic applications, such as optical filters, metamaterials, polarizers or switches. ${ }^{17-24}$ The large birefringence combined with versatile driving methods for switching make LC-NP composites an attractive material system for reconfigurable active plasmonic devices. ${ }^{25}$ The switching of such composites was recently experimentally demonstrated for $\mathrm{Ag}$ and $\mathrm{Au}$ NPs where structural changes of the LC-NP composite were induced by a thermal stimulus and led to a tunable plasmonic response. ${ }^{26,27}$ A number of studies report on the successful co-assembly of NPs by LCs into geometries that mimic the mesogen arrangement. ${ }^{28-32}$

Solubilising such nanometer-sized objects in a LC is, however, a known and widespread challenge. ${ }^{33}$ The dense periodic packing of LC mesogens frustrates mixing and free diffusion of additives. ${ }^{34}$ To-date, the most successful strategies to solubilize NPs rely on creating a ligand shell that resembles the mesogen environment, which is typically achieved by a mixture of mesogen-like ligands and shorter ligands that serve as spacers and thus create supramolecular pockets for mesogen penetration. ${ }^{35,36}$ This approach has made it possible to produce LC-NP composites with NP loadings exceeding $50 \mathrm{wt} \%{ }^{37}$ At significant NP loadings, the presence of non-nematogenic species was found to have profound effects on the phase behavior of nematic LCs, and several theoretical and experimental studies have established phase diagrams of LCNP composites. ${ }^{37-39}$ Exploitation of liquid crystal phase transition processes offers access to structure formation beyond the nanometer length scale. ${ }^{40-44}$ The presence of an isotropic-nematic co-existence region above a critical NP threshold enabled the formation of macroscopic NPrich networks. ${ }^{45}$ Recently, NP-based hollow microstructures were formed through a two-stage nematic nucleation process. ${ }^{46}$ In a separate study, cooling of LC-NP composites allowed the spatial separation of quantum dot clusters; however, control over the positional order was limited and only achieved by the introduction of macroscopic beads as nucleation points. ${ }^{47}$

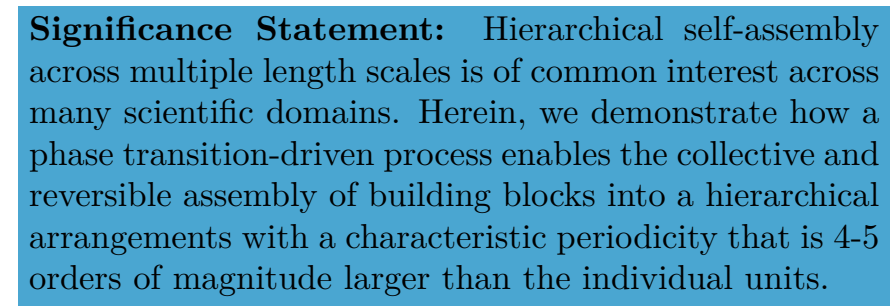

\footnotetext{
${ }^{1}$ Department of Chemical Engineering, University College London, Torrington Place, London, WC1E 7JE, UK. ${ }^{2}$ Institute of Materials, École Polytechnique Fédérale de Lausanne, 1015 Lausanne, Switzerland. ${ }^{3}$ Department of Applied Physics, Eindhoven University of Technology, Eindhoven, The Netherlands. ${ }^{4}$ Department of Materials Science and Engineering, Massachusetts Institute of Technology Cambridge, Massachusetts 02139, USA. ${ }^{\dagger}$ Now at: Adolphe Merkle Institute, Chemin des Verdiers, 1700 Fribourg, CH. *e-mail: S.Guldin@ucl.ac.uk
} 
In this work, we present how the temperature-induced isotropic-to-nematic phase transition of the thermotropic LC 4'-pentyl-biphenyl-4-carbonitrile (5CB) can drive a hierarchical assembly of nanometer-sized gold particles into micrometer-sized aggregates. This process is not only reversible but offers control over the characteristic size and spacing of the resulting structures. We report on the dynamics of this process and correlate our findings to parameters such as the cooling rate, nanoparticle solubility, phase separation kinetics, and the properties of the nematic director field.

\section{Results \& discussion}

$\mathrm{Au}$ NPs were made in a modular approach, which allowed independent control over both the NP size and ligand composition on the surface. ${ }^{48}$ First, oleylamineprotected NPs were synthesized and these were subsequently functionalized by ligand exchange with a 60:40 mol\% mixture of 1-hexanethiol (HT) and 4'(12-mercaptododecyloxy)biphenyl-4-carbonitrile (MDD$\mathrm{CBO}$ ), which led to an effective ligand composition on the NP surface of $61: 39 \pm 3 \mathrm{~mol} \%$ according to analysis by NMR spectroscopy. The Au NP size distribution was $4.7 \pm 0.7 \mathrm{~nm}$ according to TEM analysis. Another batch of Au NPs with a size distribution of $6.0 \pm 1.0 \mathrm{~nm}$ and comparable ligand composition was prepared that yielded results similar to those reported here. The NPs were then transferred to $5 \mathrm{CB}$ in the isotropic phase at a concentration of $5 \mathrm{wt} \%$ following an established protocol using dichloromethane as a volatile solvent. ${ }^{49}$ Subsequently, the LC-NP composite was infiltrated into a LC cell with defined gap thickness of $20 \mu \mathrm{m}$ and homogeneous surface alignment. Further details on the NP synthesis, cleaning procedure, solubility, and material characterisation can be found in the Supplementary Information (Supplementary Figs. S3-S9). Interestingly, the NPs showed superior solubility in isotropic $5 \mathrm{CB}$ compared to various common solvents, including chloroform, chlorobenzene, and a $50 / 50 \mathrm{vol} \%$ mixture of acetonitrile and tetrahydrofuran (Supplementary Fig. S10).

The dynamics of the LC phase transition and subsequent diffusion of NPs into the nematic phase are shown in Fig. 1. The LC-NP composite was initially heated at
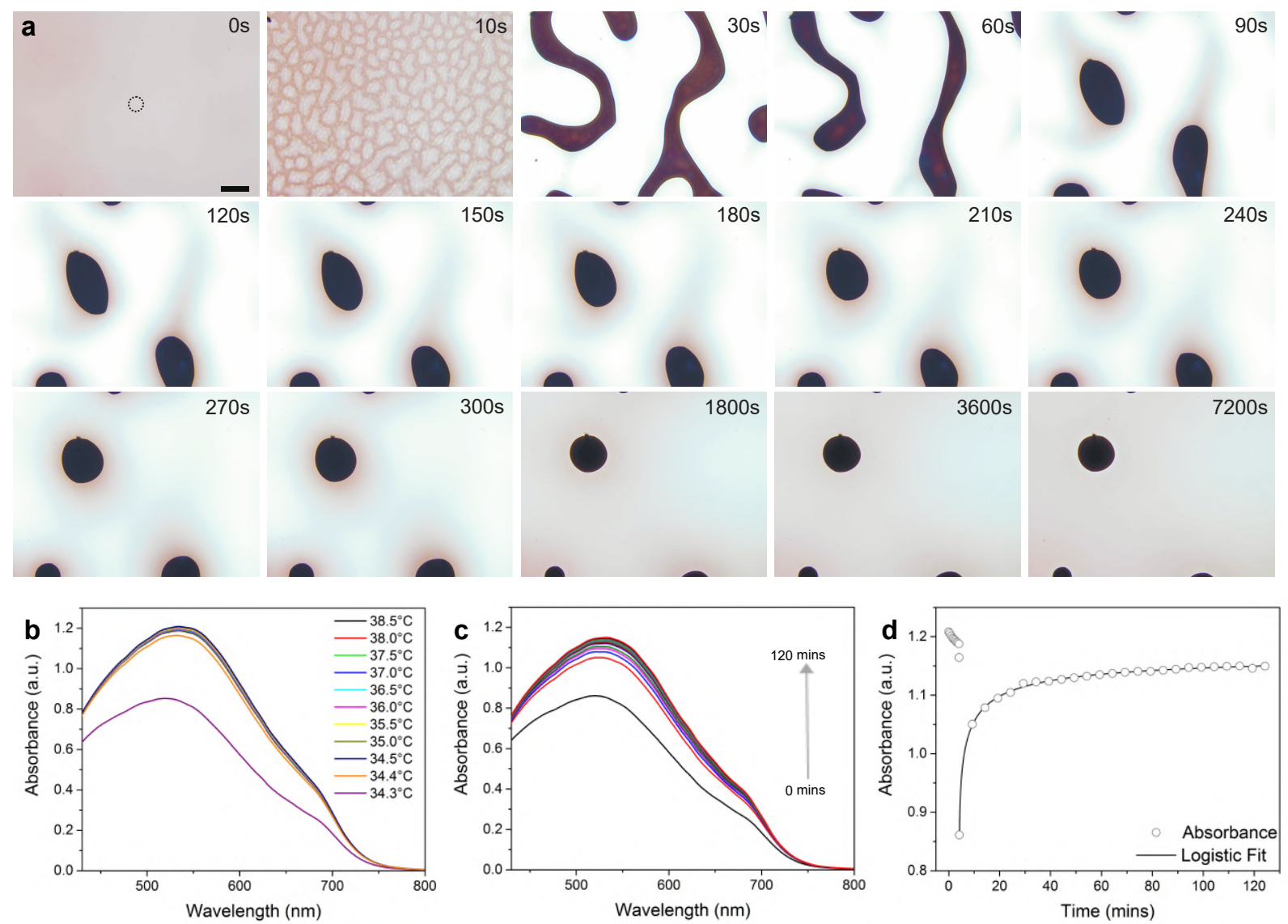

Figure 1 | Liquid crystal phase transition and dynamics of nanoparticle diffusion into the nematic liquid crystal. a, Kinetic series of bright field microscopy images of liquid crystal - nanoparticle composite upon cooling to $34.3^{\circ} \mathrm{C}\left(1^{\circ} \mathrm{C} / \mathrm{min}\right)$. Images were acquired with a 20x objective. The scale bar represents $50 \mu \mathrm{m}$. b-d, Absorbance microspectroscopy was carried out during this assembly process on a $25 \mu \mathrm{m}$-sized collection spot, represented by the dotted circle in a. In-situ absorbance spectra are plotted during cooling at $1{ }^{\circ} \mathrm{C} / \mathrm{min}$ from 38.5 to $34.3^{\circ} \mathrm{C}$ (b) and then at a constant $34.3^{\circ} \mathrm{C}$ with 5 min intervals (c). d, $A b s_{\max }$ vs. time from 0 to $120 \mathrm{~min}$, corresponding to the image series shown in $\mathbf{a}$. 
$50{ }^{\circ} \mathrm{C}$ for two hours and subsequently cooled at a rate of $1^{\circ} \mathrm{C} / \mathrm{min}$ until a phase transition was observed, which occurred at $34.3^{\circ} \mathrm{C}$. A series of images was then taken at this temperature alongside microspectroscopic absorbance measurements by partial out-coupling of light. By analysis of the micrographs, the behavior observed upon cooling may be divided into two stages. In the initial stage within the first $60 \mathrm{~s}$, a compartmentalisation occurred into NPdepleted and NP-rich isotropic that resembled a spinodaltype decomposition; over time, the characteristic continuous pattern evolved to yield isolated NP aggregates at the end of the process. Cross-polarized optical microscopy revealed that the regions which appeared brighter under bright field illumination in the initial decomposition phase corresponded to nematic domains, while the dark regions were isotropic (see Supplementary Figs. S11-S12).

The findings of in-situ absorbance microspectroscopy are shown in Fig. 1b-d. Note that the absorbance of the $5 \mathrm{CB}$ mesogens within the $20 \mu \mathrm{m}$ optical path is negligible in both the nematic and isotropic state and absorbance microspectroscopy hence offers a viable route to study the concentration of NPs in this assembly process with a $\mu \mathrm{m}-$ sized spatial resolution. Above the isotropic-to-nematic phase transition temperature, gradual cooling did not result in significant changes in the maximum absorbance of the LC-NP composite. However, once the phase transition was triggered at $34.3^{\circ} \mathrm{C}$, the maximum absorbance $\left(A b s_{\max }\right)$ decreased drastically from 1.21 to 0.86 in the newly formed nematic domains (Fig. 1b). Absorbance spectra were subsequently acquired at this temperature in 5 min intervals. As shown in Fig. 1c and related microscopy images, a rapid increase in absorption to $A b s_{\max }$ $=1.05$ within the first $5 \mathrm{~min}$ and a further increase to $A b s_{\max }=1.15$ was observed over time, which can be fitted by a logistic growth function (Fig. 1d). Based on experiments at concentrations below $1.5 \mathrm{wt} \%$, where no residual aggregates formed, a similar molar attenuation coefficient was obtained for the LC-NP composite in the isotropic and nematic phase, respectively (Supplementary Fig. 13). The absorbance results are thus in line with a short-lived depletion of NPs in the newly formed nematic domains followed by subsequent diffusion of NPs from the enriched receding isotropic into the nematic phase. After reaching equilibrium conditions, the large majority of the NPs were dispersed in the nematic phase with the residual fraction of NPs embedded as aggregates in the LC-NP nematic matrix.

To examine underlying effects, we developed a qualitative coarse-grained molecular dynamics model of a single NP complex with mixed ligand shell immersed in a host of nematogenic mesogens, which is discoursed in detail in the Supplementary Information (Supplementary Figs. S1-S2). We find that while interdigitation of the ligand shell generally facilitates solvation of the NP complex, the solvation free energy depends on whether the medium is isotropic
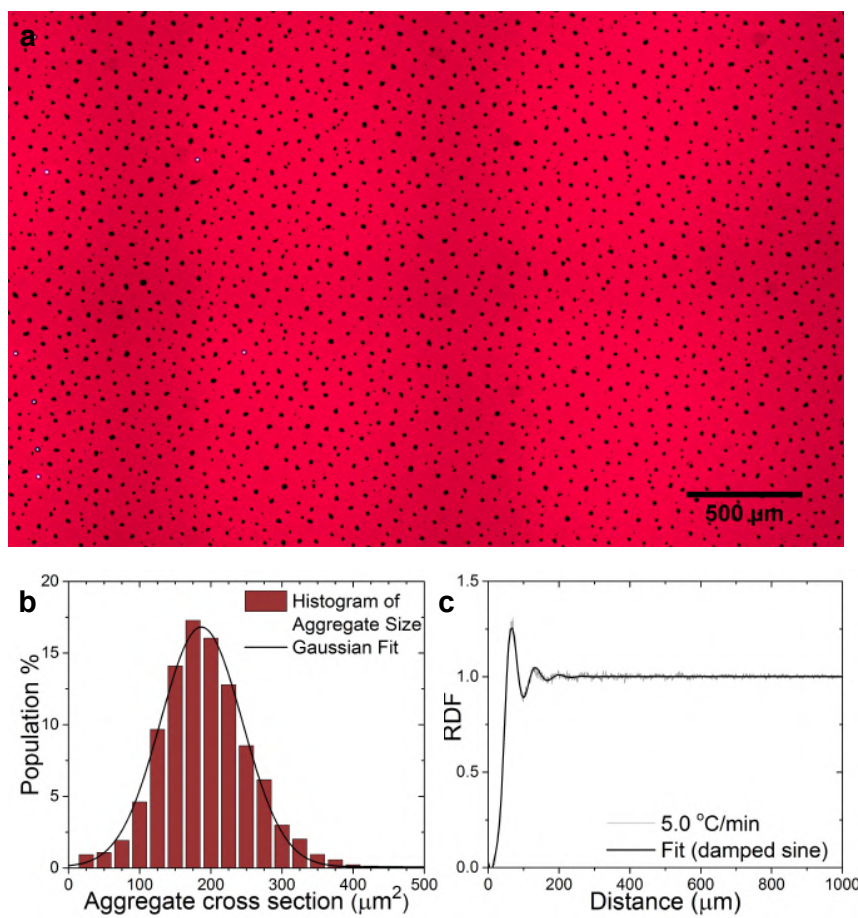

Figure 2 | Long-range nanoparticle aggregate formation. a, Bright field transmission microscopy image of nematic liquid crystal - nanoparticle composite at $28{ }^{\circ} \mathrm{C}$ that was cooled from the isotropic phase at a rate of $5^{\circ} \mathrm{C} / \mathrm{min}$. b. Size distribution of the aggregates and $\mathbf{c}$, radial distribution function of the aggregate positions (center point) fitted with a damped sine function.

or nematic. In the nematic phase, solvation of an object requires distorting the local order parameter, which introduces a free energy penalty to the system. A solvation barrier as driving force for a phase transition-driven collective assembly may be conceptualized as follows. Cooling of the LC-NP composite to the isotropic-to-nematic phase transition temperature $T_{\text {iso-nem }}$ induces demixing and a shortterm phase co-existence of NP-enriched isotropic and NPdepleted nematic regions. The build-up in NP concentration in the receding isotropic phase then forces additional NPs to slowly diffuse into the nematic phase until, eventually, micrometer-sized aggregates of residual NPs are retained.

Our findings are consistent with earlier studies on the behavior of larger hard sphere colloids in thermotropic liquid crystals. Petrov and Terentjev proposed a mechanism by which particles smaller than the surface extrapolation length, $\lambda=K / W$, form cellular networks upon cooling from the isotropic to the nematic. ${ }^{50,51}$ In the vicinity of the weak first order phase transition, $K$ and $W$ approach 0 at different rates. This would result in a decrease in the ratio $K / W$ and a corresponding increased elastic distortion due to the colloidal inclusions and expulsion of the particles from the nematic domains. Poon, Terentjev and coworkers showed that 100-1000 nm PMMA particles were expelled from nematic nuclei during cooling from the isotropic phase, ultimately forming network-type struc- 
tures with characteristic length scales. ${ }^{40,41,50,51}$ Faster cooling rates resulted in a greater number of nematic domains which produced a more tightly packed network. Recently, Abbott and coworkers demonstrated that such networks can be formed via a two step process involving an initial spinodal decomposition of the colloidal dispersion in the isotropic phase followed by the nucleation of nematic domains. ${ }^{42}$

In Fig. 2a, a long-range bright transmission microscopy image of a nematic composite after cooling at a rate of $5{ }^{\circ} \mathrm{C} /$ min to $28^{\circ} \mathrm{C}$ is shown. In order to determine the characteristic spacing between the aggregates and evaluate ordering, the radial distribution function (RDF) was calculated. Rastering acquisition with a $5 \mathrm{X}$ objective allowed analysis of the entire active area of the LC cell $(10 \times 10 \mathrm{~mm})$, which contained between 1,500 and 60,000 aggregates depending on the cooling rate. ${ }^{52}$ We refer to the Supplementary Information for a comprehensive comparison of the RDF with the nearest neighbor distance (NND) approach, in particular on their robustness towards small positional deviations (Supplementary Fig. S14). As shown in Fig. 2c, the nanoscale building blocks formed a pattern of $\mu \mathrm{m}$-scale aggregates with characteristic interaggregate distances of $68 \pm 2 \mu \mathrm{m}$, evidenced by a periodic fluctuation of the RDF that can be fitted with a modified damped sine function.

We want to emphasize both the stability of the aggregates upon formation and the reversibility of the process. Once formed, the aggregates in the nematic phase were found to be stable for months only to be redissolved by heating to temperatures above the isotropic phase transition. To quantify the reversibility, i.e. whether the assembly would fully dissolve upon heating to the isotropic state, each image of the composite, comprising an area of approximately $3000 \mu \mathrm{m}^{2}$, was converted to a grayscale matrix. The information entropy was then calculated and normalized against the image area (see Figure 3b). ${ }^{53,54}$ For a perfectly homogenous system, each pixel value should be equivalent, resulting in a maximum entropy per $\mu \mathrm{m}^{2}$ of 1 . As variation increases, the entropy is reduced. Due to the presence of the glass spacer beads and other minor aberrations in the LC cell and optical path, the maximum cannot be achieved but the reversibility over time can be tracked. Prior to the phase transition, the entropy per $\mu \mathrm{m}^{2}$ remained relatively constant at an average of $0.979 \mu \mathrm{m}^{-2}$. At the onset of the nematic to isotropic transition, this dropped drastically to $0.828 \mu \mathrm{m}^{-2}$. With diffusion of the AuNPs into the isotropic, the entropy per $\mu \mathrm{m}^{2}$ increased towards 1 following a logistic trend, demonstrating homogeneity.

In order to capture the phenomenology presented in Fig. 1 , we modeled the morphological evolution of the phase transition and NP segregation with coupled Allen-Cahn and Cahn-Hilliard equations. The Allen-Cahn dynamics were chosen for non-conserved order parameters; in this
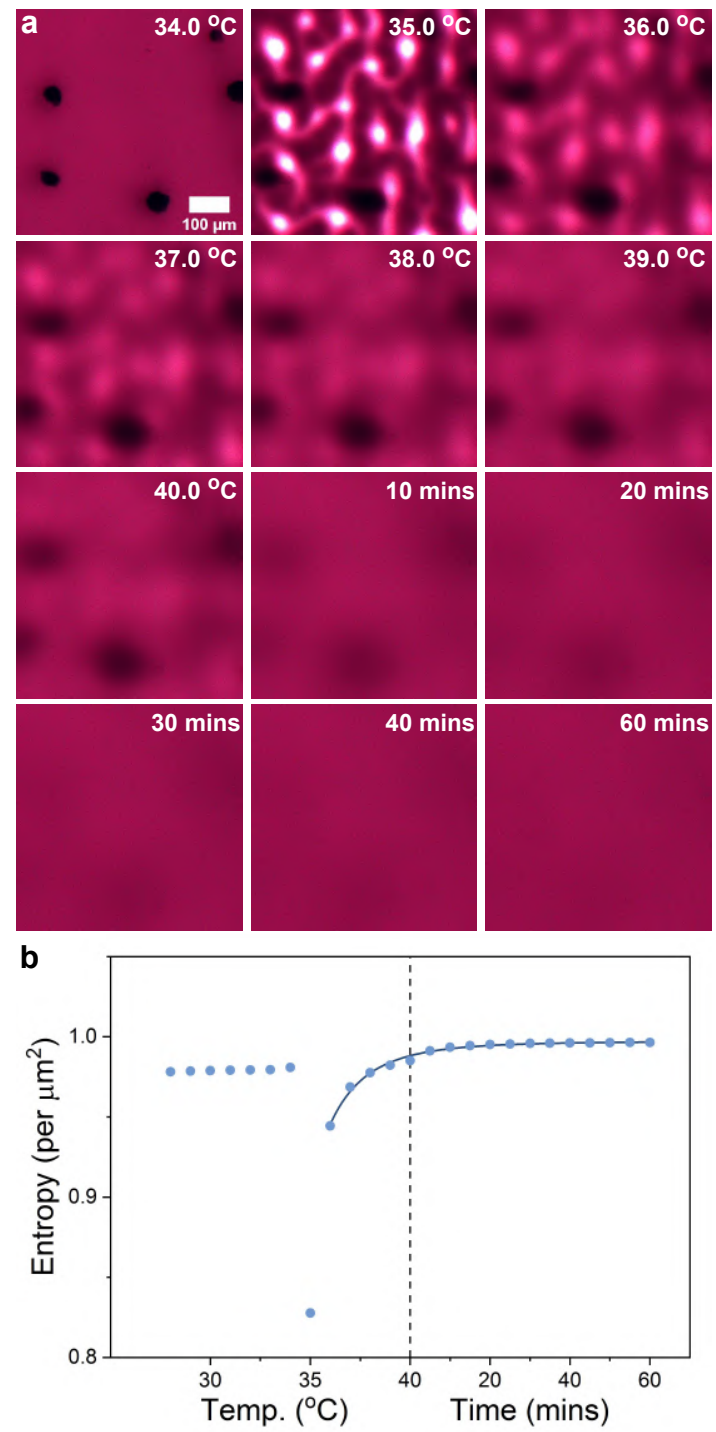

Figure $3 \mid$ Reversibility of aggregate array formation. a A series of kinetic bright field microscopy images of the composite heated at $1 \% / \mathrm{min}$ through the phase transition to $40^{\circ}$ and held for 60 mins. b The information entropy was used to evaluate the homogeneity of the composite as it is heated and maintained in the isotropic phase.

case, the magnitude of the LC director field, $\eta(\vec{x})$, was taken to be zero in the isotropic region and one in the completely ordered LC phase. Boundaries that divide one LC orientation from another were not modeled. The CahnHilliard dynamics were used for conserved order parameters; in this case the composition, $c(\vec{x})$ which was taken to be one when the system was pure mesogen and zero when the system was pure NP. Cahn-Hilliard dynamics allowed $c(\vec{x})$ to vary while preserving the system's average composition The coupling of $c(\vec{x})$ and $\eta(\vec{x})$ occured via a phenomenological free-energy function, $f(c, \eta)$ such as that illustrated in Fig. $4 \mathrm{~g}$ where composition and order are plotted along the horizontal- and vertical-axes and 

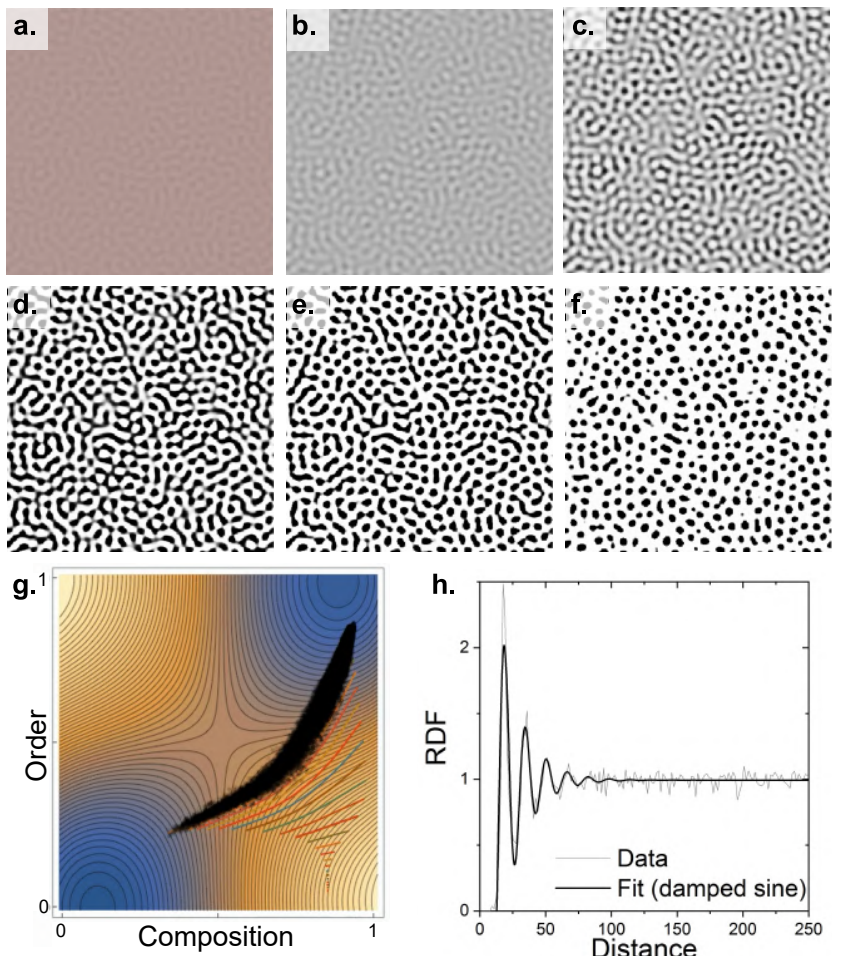

Figure 4 Simulation of NP segregation and aggregate ordering. a-f Sequential snapshots of the evolving microstructure. The black-and-white contrast corresponds to the composition, where black/white indicates high/low NP concentration. The pink color indicates the degree of order and is scaled to accentuate contrast for more disordered states. g Morphology evolution superimposed on a free-energy landscape, where the horizontal and vertical axes are NP composition and degree of order, respectively. The freeenergy landscape was empirically chosen so that minima are located at (i) low-NP composition and low-order and (ii) high NP composition and high-order. h Radial distribution functions obtained by the simulation and an example experiment.

the free-energy density is represented by isocontours. The locations of the maxima, minima, and saddle points of $f(c, \eta)$ were chosen to be consistent with the observations presented above as described in Fig. 4's caption.

The equilibrium compositions are given by the common tangent construction on the projection of the free energy surface $f(c, \eta)$ onto a constant $\eta$-plane-or, equivalently the common tangent construction on the curve $\left.f(c) \equiv \min _{\eta} f(c, \eta)\right) .{ }^{55}$ The system will spontaneously decompose into NP-rich and NP-depleted regions at any composition and order parameter where at least one of the principle curvatures of the surface $f(c, \eta)$ is negative-this is spinodal decomposition. The "direction" of decomposition in the $c-\eta$ plane (i.e., the orientation of the growing curve in Fig. 4) is restricted to those directions that have a negative curvature; the specific direction depends on that curvature and the ratio of the two time constants (i.e., if the time constant for LC ordering is fast, then the developing curve in Fig. 4 will tend towards being vertical but moderated by the curvature in that direction).
If sufficiently large order and composition fluctuations are present, then the system will continue to decompose at any composition that lies above the common tangent-that is, some initial spinodal decomposition can produce a surface that acts as a supercritical nucleus.

The simulations illustrated in Fig. 4 can be interpreted as follows. At $t=0$, the disordered $(\eta \approx 0)$ system begins to decompose into slightly NP-enriched (towards the right) and depleted compositions. The regions that have depleted compositions experience a larger driving force to order and therefore the decomposition produces a concomitant order-disorder transition with the more isotropic regions being slightly enriched. This initial decomposition can be observed in Fig. 4(a-b) and has the morphology of a spinodal decomposition. The system's equilibrium compositions and phase fractions are determined by the common tangent construction described above; therefore the final state of the system will be mostly the NPdepleted LC phase and a small amount of isotropic NPrich phase. Thus, the final state cannot have the bothphase-percolating (e.g., "brain-like") morphology that is commonly associated with a $X_{A}=X_{B}=1 / 2$ composition for the regular solution model. Instead, the initial fluctuations must break the minor phase into isolated regions-this produces a structure that is more commonly associated with nucleation and growth; but in this case, the structure is initiated by a decomposition reaction. The shape of the developing curve gives co-variation of composition and order across the interface. Most of the material is located at the ends of the developing curve. The curve only represents the interface and so most of the visual data in Fig. 4 represent a small part of the microstructure.

The developing curve should evolve toward a fixed curve that minimizes the surface tension in the system. Presumably this minimizing curve should pass near the saddle point of $f(c, \eta)$ but is also influenced by the square gradient coefficients. The full analysis of the direction of initial composition and the equilibrium interface is beyond the scope of this paper. The initial decomposition produces a large amount of interface. At small times, points in Fig. 4 come from a multitude of interfaces. At larger times, the system coarsens and removes many of the interfaces. It is possible to construct a free-energy function for which the final microstructure is LC with solubilized NPs-and which undergoes an initial decomposition reaction. In other words, if the free energy function has regions of negative curvature for small values of $\eta$, it is possible for the system to undergo a transient spinodal composition. As the system continues to order, the NP-rich regions will dissolve and the system will evolve towards its commontangent compositions.

The outlined characteristics of structure formation by phase transition are significantly different to conventional spinodal decomposition-based processes that exhibit a conserved order parameter, such as the demixing of poly- 

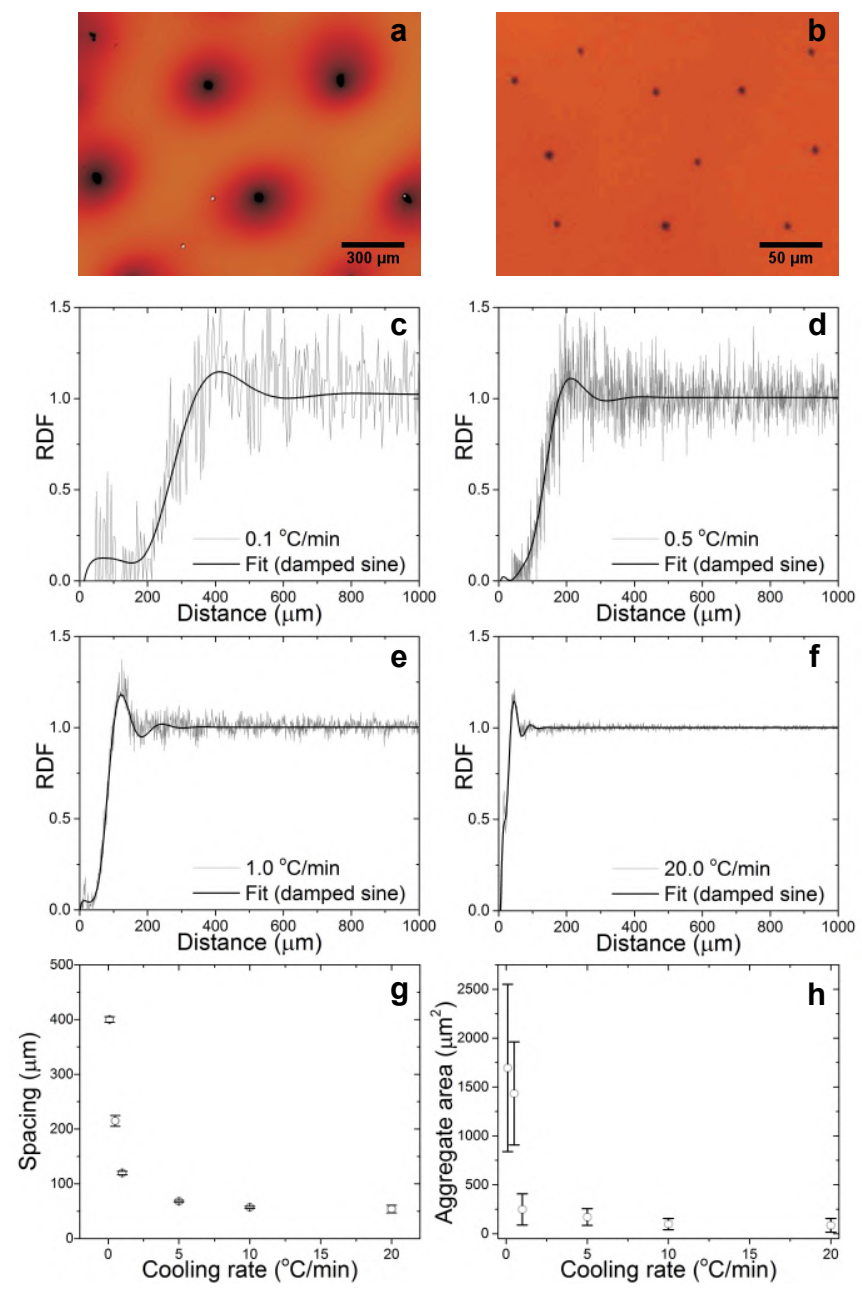

Figure 5 Effect of cooling rate on order parameters. a-b, Zoom-in of aggregates resulting from a cooling rate of $0.1^{\circ} \mathrm{C} / \mathrm{min}$ and $20^{\circ} \mathrm{C} / \mathrm{min}$, respectively. c-f, Radial distribution function and corresponding damped sine fit for liquid crystal - nanoparticle composites exposed to different cooling rates. f, Overview of characteristic spacing as a function of cooling rate. $\mathbf{g}$, Overview of mean aggregate size as a function of cooling rate.

mer blends ${ }^{56,57}$, sol-gel-polymer ${ }^{58}$ and fullerene-polymer composites $^{59}$, polyelectrolyte multilayers or the electrochemical or liquid metal dealloying. ${ }^{60,61}$ The approach presented here starts with a homogeneous mixture that is fully isotropic. Cooling induces demixing with NPenriched isotropic and NP-depleted nematic regions. The phase co-existence is only transient and thus, at the end of the process, the composite displays the nematic phase with a characteristic spacing and size of aggregates depending on the cooling rate.

The effect of the cooling protocol on the aggregate ordering parameters is shown in Fig. 5. Six different cooling rates, ranging from $0.1^{\circ} \mathrm{C} / \mathrm{min}$ to $20^{\circ} \mathrm{C} / \mathrm{min}$ were studied. Samples were cooled to $28^{\circ} \mathrm{C}$ and left to equilibrate for two hours. Between cycles, the LC-NP composite was heated to $50{ }^{\circ} \mathrm{C}$ for two hours to erase the sample history, and the process was repeated multiple times to evidence full reversibility. The corresponding RDFs demonstrate a non-linear decrease in inter-aggregate spacing with increasing cooling rate. Below $1^{\circ} \mathrm{C} / \mathrm{min}$, a small increase in the cooling rate produced a significant reduction in the average inter-aggregate distance. Higher cooling rates, in contrast, exhibited a much diminished dependence. Likewise, the mean aggregate size was found to depend on the cooling rate as shown in Fig. 5h and in the Supplementary Fig. 15. Note that the increased noise for slower cooling rates is related to the significantly reduced population size considered in the RDF, because fewer aggregates were formed and these were spaced further apart. In this series of experiments, an absorbance of 1.36 was found for the LC-NP composite in the isotropic state, which was reduced to values of 1.32 to 1.24 in the nematic state for cooling rates ranging from $0.1^{\circ} \mathrm{C} / \mathrm{min}$ to $20^{\circ} \mathrm{C} / \mathrm{min}$ (Supplementary Fig. 16). The higher $A b s_{\max }$ value indicates that lower cooling rates also led to an overall improved transfer rate of $\mathrm{Au}$ NPs from the isotropic to the nematic phase.

The dynamics of the assembly process for different cooling rates was further studied by time-lapse microscopy. Though the principles of aggregate formation were found to be similar to the observations reported in Fig. 1, the process differed not only in the characteristic length scale but also in the characteristic time scale. While the formation of aggregates was completed in $1.5 \mathrm{~min}$ for a cooling rate of $20^{\circ} \mathrm{C} / \mathrm{min}$, the whole process required around $100 \mathrm{~min}$ at $0.1^{\circ} \mathrm{C} / \mathrm{min}$. A comparison of the spinodaltype patterns before break-up of the percolation for the receding NP-enriched isotropic phase is shown in the Supplementary Fig. 17 for different cooling rates. The observed characteristic length scale is very closely in line with the characteristic aggregate spacing after completion of the isotropic-to-nematic phase transition, demonstrating that the NP aggregates are indeed a remnant of the initial phase separation and can therefore be controlled through parameters that govern this process. ${ }^{62}$

The concentration of NPs in the isotropic phase played an important role in the overall aggregate formation. As shown in the Supplementary Fig. 18, reducing the NP concentration down to $2.5 \mathrm{wt} \%$ led to a decrease in the mean aggregate size and a less pronounced peak in the RDF. At concentrations below $1.5 \mathrm{wt} \%$, no $\mu \mathrm{m}$-sized aggregates were observed in the final nematic composite, most likely due to the fact that the transient local increase in NP concentration in the receding isotropic phase was eventually consumed by NP diffusion into the nematic phase. Furthermore, the extent of the diffusion into the bulk depended on the initial concentration. For $5 \mathrm{wt} \%$ and a cooling rate of $0.5^{\circ} \mathrm{C} / \mathrm{min}$, the bulk concentration was found to be $4.7 \mathrm{wt} \%$. At an initial concentration of $4.5 \mathrm{wt} \%$, this dropped to $4.3 \mathrm{wt} \%$. This relationship predicted that all aggregates should vanish at an AuNP content of $2.2 \mathrm{wt} \%$ which agreed well with the experimental results. Note that 

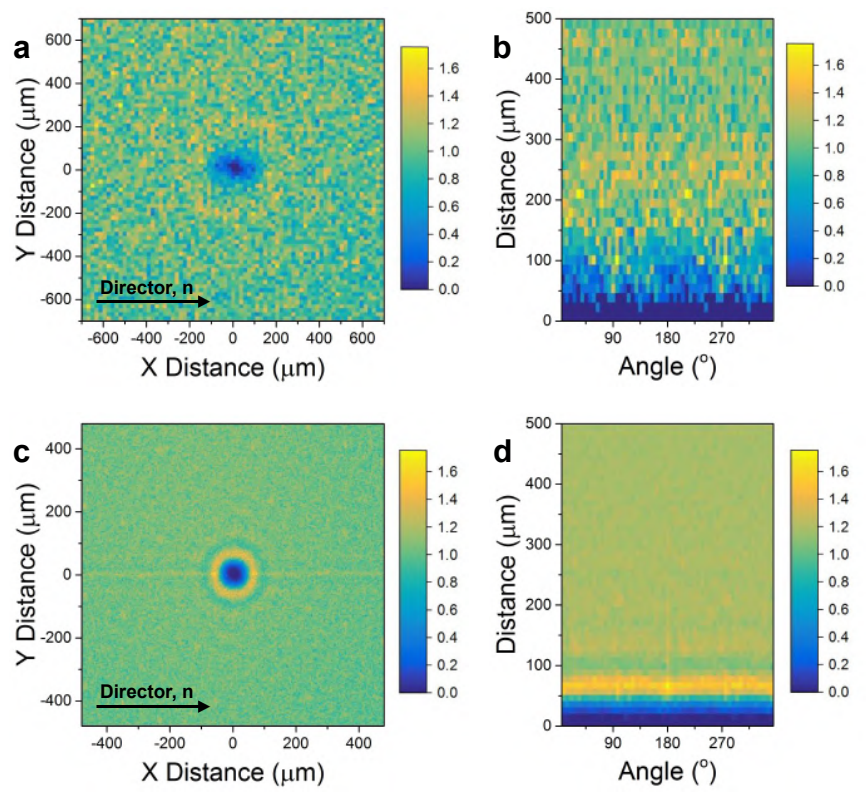

Figure $6 \mid 2 D$ orientation of nanoparticle aggregates in the liquid crystal - nanoparticle composite. $2 \mathrm{D}$ histogram of the aggregate spatial distribution for a cooling rate of $0.5^{\circ} \mathrm{C} / \mathrm{min}$ in $x-y$ coordinates (a) and polar coordinates (b), respectively. c-d, $2 \mathrm{D}$ histogram of the aggregate spatial distribution for a cooling rate of $5^{\circ} \mathrm{C} / \mathrm{min}$.

doping of the LC with Au NPs also led to a small decrease in the observed transition temperature from $34.8^{\circ} \mathrm{C}$ for $0 \mathrm{wt} \%$ to $34.3^{\circ} \mathrm{C}$ for $5 \mathrm{wt} \%$, which is in line with previous studies that reported on a NP-induced dilution effect. ${ }^{37,39}$ Further details on the effect of NP concentration and applied cooling rate on the observed phase transition temperature can be found in Supplementary Figs. S19-S20.

The most important consequence of this process is the hierarchical organisation of NPs into larger assemblies within a nematic composite. While the individual $5 \mathrm{~nm}$ sized NPs are too small to be recognized by the LC director field, the larger NP aggregates should indeed be subject to elastic distortion. ${ }^{16}$ The RDF demonstrates positional order of aggregates over the full radial profile and does, therefore, not account for a possible anisotropy due the nematic phase and alignment along a common director. In Fig. 6 2D histograms of the spatial distribution are shown to represent the aggregate spacing in $\mathrm{x}-\mathrm{y}$ and polar coordinates for $0.5^{\circ} \mathrm{C} / \mathrm{min}$ and $5^{\circ} \mathrm{C} / \mathrm{min}$, respectively. Details of the applied methodology, ${ }^{63}$ the experimental results for other cooling rates as well as a simulation of the effect of disorder on the resulting $2 \mathrm{D}$ histograms can be found in Supplementary Figs. S21-S22. At $0.5^{\circ} \mathrm{C} / \mathrm{min}$ (Fig. 6a), i.e. for larger aggregates that are further spaced from each other, the aggregate depletion zone (the region in which the likelihood of finding another aggregate is below the mean) exhibited an ellipsoidal shape and thus a clear anisotropy. In order to measure the eccentricity of this behavior, an angular window was chosen that allowed sufficient statistical evidence for the determination of the characteristic spacing by a segmented radial profile, here $10^{\circ}$. The segmented radial profile along the director field was determined and compared with the segmented radial profile of the respective angular window perpendicular to the director field. Based on the characteristic spacing obtained in both directions, an eccentricity of $0.71 \pm 0.02$ was found. The spatial modulation as a function of the relative orientation to the mesogen director field is directly evident in the polar coordinate plot shown in Fig. $6 \mathrm{~b}$. At higher cooling rates and thus smaller aggregates with smaller spacing $\left(5^{\circ} \mathrm{C} / \mathrm{min}\right.$ to $\left.20^{\circ} \mathrm{C} / \mathrm{min}\right)$, an anisotropy in the depletion zone was not observed. Instead, an increased probability of finding aggregates along the director field became evident, observable in Fig. 6c as a yellow line along the $Y=0$ direction and in Fig. $6 \mathrm{~d}$ as an increased probability around the $180^{\circ}$ window. A segmented radial profile over an angular window of $1^{\circ}$ in the direction of director field was compared to that around $45^{\circ}$ and $90^{\circ}$ with respect to the direction of the director field. Based on the results obtained herein for a cooling rate of $5^{\circ} \mathrm{C} / \mathrm{min}$, the probability of finding another aggregate along the director field was $9 \%$ higher than in any other direction. A similar trend was observed for cooling rates of $10^{\circ} \mathrm{C} / \mathrm{min}$ and $20^{\circ} \mathrm{C} / \mathrm{min}$.

A common form of colloidal assembly in nematic LCs is chain formation, typically associated to topological defects that originate from the surface anchoring conditions of mesogens. ${ }^{7}$ The resulting elastic dipoles and quadrupoles decay with distance as $1 / l^{3}$ and $1 / l^{5}$, respectively. ${ }^{64,65}$ The herein described assembly process separates the aggregates evenly over distances that are significantly larger than the mean aggregate sizes. We determined for spacing $l$ and diameter $d$ the dimensionless ratio to be in the range of $8<l / d<18$. At such separation distances, chain formation through elastic multipoles is not expected. Indeed, the formation of dimers was only a very rare event and no evidence for trimers or longer chains was found.

Finally, we must emphasize the role of the homogeneous substrate, which imposes an in-plane alignment of the mesogen director field. As shown in Supplementary Fig. S23, we did not observe the formation of aggregates with pronounced characteristic spacings when using LC cells with homeotropic, i.e. vertical, alignment. This is in line with a study by Reven and coworkers, who investigated NP ordering by 4'-Octyl-biphenyl-4-carbonitrile (8CB). ${ }^{66}$ At the nematic-smectic A phase transition, NPs were found to decorate the edge dislocation defect lines, while no characteristic nearest neighbor distance was identified at the isotropic-nematic transition. Furthermore, when we used cells without surface alignment, cooling of the LC-NP composite led to the formation of NP-rich and $\mathrm{NP}$-depleted regions but these remained in co-existence (see Supplementary Fig. S24), which is in line with earlier findings. ${ }^{39,45}$ 


\section{Conclusions}

In summary, we report on the reversible self-organized collective assembly of gold NPs in a thermotropic LC. Based on our observations, the phase-dependent solubility and the cooling temperature play a decisive role in the formation of hierarchically structured LC-NP composites comprising NP aggregates of controllable size and characteristic spacing. While reduced solubility in the nematic phase results in residual NPs to be expelled in aggregates, their spacing depends on the cooling rate that defines a characteristic length scale in the compartmentalisation through the imposed transient decomposition process during phase transition. Once formed, the aggregates were found to be stable for months and the process remained reversible by heating above the nematicto-isotropic phase transition temperature. This observation of controlled and phase transition-driven reversible assembly of gold NPs takes inspiration from other forms of stimuli-directed self-assembly, ${ }^{67}$ where the stimuli may be a redox reaction, solvent addition, $\mathrm{pH}$ change, or light exposure. ${ }^{68,69}$ The presented findings of such a controlled assembly process may yield interesting opportunities towards programmable composites for metamaterials, ${ }^{70}$ in sensing and display applications. Furthermore, we anticipate this to be an interesting synthetic model system for the further exploration of active soft matter. ${ }^{71-74}$

\section{Methods}

Ligand synthesis. The ligand synthesis was carried out following an adapted literature procedure from Milette and coworkers. ${ }^{36}$

General Procedures: Unless otherwise noted, all reactions were carried out in dried Schlenk glassware in an inert argon atmosphere. Chromatography solvents were purchased as reagent grade and distilled once prior to use. For reactions, dichloromethane (DCM), methanol (MeOH), tetrahydrofuran (THF), and $N, N$ dimethylformamide (DMF) were purchased dry over molecular sieves from Acros Organics, and acetone was purchased dry from Sigma-Aldrich. All reagents were commercially obtained and used without further purification. 4'-Hydroxy-4-biphenylcarbonitrile $(99 \%)$ and hexamethyldisilthiane $(97 \%)$ were purchased from ABCR, 1,12-dibromododecane (98\%) was purchased from TCI, potassium thioacetate (98\%) was purchased from Alfa Aesar, and acetyl chloride (ACS reagent grade), anhydrous potassium carbonate $(99 \%)$, and tetrabutylammonium fluoride (1 M solution in THF) were purchased from Acros Organics. TLC analyzes were performed on TLC plates from Merck (Silica gel $60 \mathrm{~F}_{254}$ ). UV-light $(254 \mathrm{~nm})$ or anisaldehyde staining was used for detection. Column chromatography was conducted on Geduran silica gel Si 60 from Merck (40-60 $\mu \mathrm{m})$.

4'-(12-Bromododecyloxy)-4-biphenylcarbonitrile 1. 4' Hydroxy-4-biphenylcarbonitrile $(4.38 \mathrm{~g}, 22.4 \mathrm{mmol})$ and $1,12-$ dibromododecane $(36.82 \mathrm{~g}, \quad 112.2 \mathrm{mmol})$ were dissolved in dry acetone $(750 \mathrm{~mL})$. Potassium carbonate $(6.11 \mathrm{~g}, 44.9 \mathrm{mmol})$ was added and the mixture was heated to reflux for $10 \mathrm{~h}$, after which 4'-hydroxy-4-biphenylcarbonitrile was consumed according to TLC (DCM). After dilution with DCM $(300 \mathrm{~mL})$ the mixture was washed twice with $1 \mathrm{M} \mathrm{HCl}$ and once with saturated $\mathrm{NaCl}$ solution. The organic phase was dried over $\mathrm{MgSO}_{4}$, and concentrated in vacuo. Column chromatography (silica gel; DCM/n-heptane 1:2) afforded 4'-(12-bromododecyloxy)-4- biphenylcarbonitrile 1 (8.69g, $19.6 \mathrm{mmol}, 88 \%)$ as a colorless powder. ${ }^{1} \mathrm{H}$ NMR $(400.13 \mathrm{MHz}$, $\left.\mathrm{CDCl}_{3}\right): \quad \delta=7.70-7.63(\mathrm{~m}, 4 \mathrm{H}, \mathrm{Ph} H), 7.54-7.51(\mathrm{~m}, 2 \mathrm{H}, \mathrm{Ph} H)$
7.01-6.97 (m, 2H, $\mathrm{PhH}), 4.01\left(\mathrm{t}, J=6.5 \mathrm{~Hz}, 2 \mathrm{H}, \mathrm{CH} \mathrm{H}_{2} \mathrm{OPh}\right), 3.41(\mathrm{t}$ $\left.J=6.9 \mathrm{~Hz}, 2 \mathrm{H}, \mathrm{CH}_{2} \mathrm{Br}\right), 1.89-1.77\left(\mathrm{~m}, 4 \mathrm{H}, 2 \mathrm{CH}_{2}\right), 1.51-1.29(\mathrm{~m}$, $\left.16 \mathrm{H}, 8 \mathrm{CH}_{2}\right) .{ }^{13} \mathrm{C} \mathrm{NMR}\left(100.61 \mathrm{MHz}, \mathrm{CDCl}_{3}\right): \delta=159.9,145.4$, $132.7,131.4,128.4,127.2,119.3,115.2,110.2(8 \mathrm{Ph} C, 1 C \mathrm{~N}), 68.3$ $\left(\mathrm{CH}_{2} \mathrm{OR}\right), 34.2\left(\mathrm{CH}_{2} \mathrm{Br}\right), 33.0,29.7,29.7,29.6,29.6,29.5,29.4$, $28.9,28.3,26.2\left(10 \mathrm{CH}_{2}\right)$

S-(12-(4'-(4-Biphenylcarbonitrile)oxy)dodecyl)

ethanethioate 2. 4'-(12-Bromododecyloxy)-4-biphenylcarbonitrile $1(0.98 \mathrm{~g}, 2.2 \mathrm{mmol})$ was added to a dispersion of $S$-potassium thioacetate $(1.25 \mathrm{~g}, 11.0 \mathrm{mmol})$ in $\operatorname{DMF}(30 \mathrm{~mL})$ and the mixture was heated to $70^{\circ} \mathrm{C}$ for $12 \mathrm{~h}$. After dilution with DCM $(150 \mathrm{~mL})$ the mixture was washed six times with $1 \mathrm{M} \mathrm{HCl}$ and once with saturated $\mathrm{NaCl}$ solution. The organic phase was dried over $\mathrm{Na}_{2} \mathrm{SO}_{4}$, and concentrated in vacuo. Column chromatography (silica gel; DCM/ $n$-heptane 5:1) afforded $S$-(12(4'-(4-biphenylcarbonitrile)oxy)dodecyl) ethanethioate 2 (0.91 g, $2.1 \mathrm{mmol}, 94 \%)$ as an off-white solid. ${ }^{1} \mathrm{H}$ NMR $(400.13 \mathrm{MHz}$, $\left.\mathrm{CDCl}_{3}\right): \delta=7.70-7.63(\mathrm{~m}, 2 \mathrm{H}, \mathrm{Ph} H), 7.54-7.51(\mathrm{~m}, 2 \mathrm{H}, \mathrm{Ph} H)$, 7.00-6.98 (m, 2H, PhH), $4.00\left(\mathrm{t}, J=6.6 \mathrm{~Hz}, 2 \mathrm{H}, \mathrm{CH}_{2} \mathrm{OPh}\right), 2.86$ $\left(\mathrm{t}, J=7.4 \mathrm{~Hz}, 2 \mathrm{H}, \mathrm{CH}_{2} \mathrm{SAc}\right), 2.32\left(\mathrm{~s}, 3 \mathrm{H}, \mathrm{CH} H_{3}\right), 1.81(\mathrm{dt}, J=$ $\left.14.6,6.7 \mathrm{~Hz}, 2 \mathrm{H}, \mathrm{CH}_{2} \mathrm{CH}_{2} \mathrm{OPh}\right), 1.60-1.52\left(\mathrm{~m}, 2 \mathrm{H}, \mathrm{CH}_{2} \mathrm{CH}_{2} \mathrm{SAc}\right)$, 1.50-1.43 (m, 2H, $\left.1 \mathrm{CH}_{2}\right), 1.35-1.28\left(\mathrm{~m}, 14 \mathrm{H}, 7 \mathrm{CH}_{2}\right) .{ }^{13} \mathrm{C} \mathrm{NMR}$ $\left(100.61 \mathrm{MHz}, \mathrm{CDCl}_{3}\right): \delta=196.2\left(\mathrm{CH}_{3} \mathrm{COS}\right), 160.0,145.4,132.7$, $131.4,128.5,127.2,119.3,115.2,110.2(8 \mathrm{Ph} C, 1 C \mathrm{~N}), 68.3$ $\left(\mathrm{CH}_{2} \mathrm{OR}\right), 30.8\left(\mathrm{CH}_{3} \mathrm{COS}\right), 29.7,29.7,29.6,29.5,29.4,29.3,29.3$, 29.0, $26.2\left(11 \mathrm{CH}_{2}\right)$.

4'-(12-Mercaptododecyloxy)-4-biphenylcarbonitrile 3.

From S-(12-(4'-(4-Biphenylcarbonitrile)oxy) dodecyl) ethanethioate 2: $\quad S$-(12-(4'-(4-Biphenylcarbo-nitrile)oxy) dodecyl) ethanethioate 2 (440 mg, $1.0 \mathrm{mmol}$ ) was dissolved in dry DCM $(7 \mathrm{~mL})$ and dry methanol $(10 \mathrm{~mL})$ was added. The mixture was stirred at room temperature, acetyl chloride $(0.5 \mathrm{~mL}, 7.0 \mathrm{mmol})$ was added dropwise, and stirring was continued for $6 \mathrm{~h}$. After dilution with DCM $(30 \mathrm{~mL})$ the mixture was washed once with saturated $\mathrm{NH}_{4} \mathrm{Cl}$ solution and once with saturated $\mathrm{NaCl}$ solution. The organic phase was dried over $\mathrm{Na}_{2} \mathrm{SO}_{4}$, and concentrated in vacuo. Column chromatography (silica gel; DCM) afforded 4'-(12mercaptododecyloxy)-4-biphenylcarbonitrile 3 (183 g, $0.46 \mathrm{mmol}$, $46 \%)$ as an off-white solid.

From 4'-(12-Bromododecyloxy)-4-biphenylcarbonitrile 1: 4'-(12Bromododecyloxy)-4-bi-phenylcarbo-nitrile 1 (5.00 g, $11.3 \mathrm{mmol})$ was dissolved in dry THF $(100 \mathrm{~mL})$ and the solution was cooled to $0^{\circ} \mathrm{C}$. Hexamethyldisilathiane $(2.85 \mathrm{~mL}, 13.56 \mathrm{mmol})$ was added dropwise to the solution, followed by dropwise addition of tetrabutylammonium fluoride $(12.4 \mathrm{~mL}, 1.0 \mathrm{M}$ in THF, $12.43 \mathrm{mmol})$. The reaction was allowed to warm up to room temperature and stirred for $2 \mathrm{~h}$. After dilution with DCM $(200 \mathrm{~mL})$ the mixture was washed twice with saturated $\mathrm{NH}_{4} \mathrm{Cl}$ solution and once with saturated $\mathrm{NaCl}$ solution. The organic phase was dried over $\mathrm{Na}_{2} \mathrm{SO}_{4}$, and concentrated in vacuo. Column chromatography (silica gel; $\mathrm{DCM} / n$-heptane 1:1) afforded 4'-(12-mercaptododecyloxy)-4biphenylcarbonitrile $\mathbf{3}(3.30 \mathrm{~g}, 8.3 \mathrm{mmol}, 74 \%)$ as colorless solid. ${ }^{1} \mathrm{H}$ NMR $\left(400.13 \mathrm{MHz}, \mathrm{CDCl}_{3}\right): \delta=7.70-7.63(\mathrm{~m}, 4 \mathrm{H}, \mathrm{Ph} H)$, 7.54-7.51 (m, 2H, $\mathrm{PhH}), 7.01-6.97(\mathrm{~m}, 2 \mathrm{H}, \mathrm{PhH}), 4.00(\mathrm{t}, J=$ $\left.6.5 \mathrm{~Hz}, 2 \mathrm{H}, \mathrm{CH}_{2} \mathrm{OR}\right), 2.52\left(\mathrm{q}, J=7.4 \mathrm{~Hz}, 2 \mathrm{H}, \mathrm{CH}_{2} \mathrm{SH}\right), 1.81$ (dt, $J$ $\left.=14.6,6.7 \mathrm{~Hz}, 2 \mathrm{H}, \mathrm{CH}_{2} \mathrm{CH}_{2} \mathrm{OR}\right), 1.64-1.57\left(\mathrm{~m}, 2 \mathrm{H}, \mathrm{CH}_{2}\right), 1.51-1.43$ $(\mathrm{m}, 2 \mathrm{H}, \mathrm{CH} 2) 1.37-1.28\left(\mathrm{~m}, 14 \mathrm{H}, 7 \mathrm{CH}_{2}\right) .{ }^{13} \mathrm{C}$ NMR $(100.61 \mathrm{MHz}$, $\left.\mathrm{CDCl}_{3}\right): \delta=160.0,145.4,132.7,131.4,128.5,127.2,119.3,115.2$, $110.2(8 \mathrm{Ph} C, 1 C \mathrm{~N}), 68.3\left(\mathrm{CH}_{2} \mathrm{OR}\right), 34.2,29.7,29.6,29.5,29.4$, 29.2, 28.5, 26.2, $24.8\left(11 \mathrm{CH}_{2}\right)$.

Nanoparticle synthesis. Oleylamine AuNPs were synthesized as follows. ${ }^{48} \mathrm{~A}$ precursor solution containing $10 \mathrm{ml}$ n-octane (Sigma Aldrich, puriss.), $10 \mathrm{ml}$ oleylamine (Acros, C18 80-90\%) and $0.25 \mathrm{mmol} \mathrm{HAuCl}_{4} \cdot 3 \mathrm{H}_{2} \mathrm{O}$ (Sigma Aldrich, 99.9+\% metals basis) was prepared and stirred under inert atmosphere at a temperature of $15^{\circ} \mathrm{C}$, which was controlled with $0.1 \mathrm{~K}$ precision. Separately, $0.25 \mathrm{mmol}$ of the reducing agent t-butylamine borane (Strem, $97 \%+$ ) was dissolved in a solvent mixture of $1 \mathrm{ml} \mathrm{n}$-octane and $1 \mathrm{ml}$ oleylamine. Subsequently, the reducing solution was injected quickly into 
the precursor solution and left stirring at for $1 \mathrm{~h}$. The oleylamineprotected AuNPs were subsequently 2x washed in ethanol (Fluka, HPLC grade) with a minimal amount of DCM (Carlo Erba, ACS grade) and subsequently redispersed in DCM (Carlo Erba, ACS grade). Ligand exchange was carried out by preparing a thiol solution containing $15 \mathrm{ml} \mathrm{DCM}$ with $0.127 \mathrm{mmol}$ of 1-hexanethiol (Alfa Aesar, 97\%+) and $0.085 \mathrm{mmol}$ of MDD-CBO, i.e. a 60/40 mol\% mixture. Subsequently, a solution with $25 \mathrm{mg}$ oleylamineprotected AuNPs and $5 \mathrm{ml}$ DCM was added and left stirring for $24 \mathrm{~h}$ at room temperature. AuNPs were cleaned by dispersing them in a $10 / 90 \mathrm{vol} \%$ mixture of DCM and acetone (Sigma Aldrich, puriss.) and subsequent precipitation by ultracentrifugation $(32,000 \mathrm{rpm}$ $1 \mathrm{~h})$. This step was repeated 3 times. A mixture of 10/10/80 vol\% tetrahydrofuran, acetonitrile (Carlo Erba, HPLC grade), and acetone (Sigma Aldrich, puriss.) was used for two subsequent cleaning cycles. We want to note that usual protocols such as the repeated precipitation in a poor solvent (here: acetone) and subsequent centrifugation (at 5,000 rpm for $10 \mathrm{~min}$ ) as well as cleaning by a Soxhlet extractor did not result in an NMR signal that is dominated by surface-bound ligands.

Sample fabrication. AuNP-LC composites were fabricated according to a protocol published by Qi et al.. ${ }^{49}$ In short, thiolprotected AuNPs were dissolved in DCM and mixed in the targeted w\% with the LC (5CB; 4-Cyano-4'-pentylbiphenyl, Synthon, 99,8\%). The mixture was stirred, then sonicated for $1 \mathrm{~min}$ before the volatile components were evaporated overnight at $60^{\circ} \mathrm{Cunder}$ a stream of nitrogen (Eppendorf ThermoMixer C). Subsequently, the solution was transferred to a vacuum oven (Heraeus Vacutherm), which was set to $50^{\circ} \mathrm{Cfor} 3$ hours to remove any remaining traces of solvent. Once complete, the sample was sealed and stored in the thermomixer at $40^{\circ}$ Cuntil use.

Subsequently, the AuNP-LC composite was infiltrated in a glass sandwich with defined gap thickness and surface functionalisation. Cells with a homogeneous or homotropic surface alignment and 420 umthickness were supplied by Instec Inc. Reference cells without surface alignment were built with pre-cleaned microscope slides where an initially $25 \mu$ mthick thermoplastic sealing film (DuPont Surlyn, Meltonix 1170-25) served as spacer. Prior to infiltration, the cell was placed on the temperature controlled stage and warmed to $40^{\circ} \mathrm{C}$. Once the cell had been heated, 10 plof the AuNPLC composite was taken from the vial and slowly deposited on the cell near the opening. Capillary action drew the composite into the cell. The cell was left at $40^{\circ} \mathrm{Cfor}$ at least 15 minutes to allow a uniform film to form inside.

Optical microscopy. Optical microscopy was carried out in transmission on an Olympus BX61. The following Objectives were used: 5x (UMPlanFI, NA 0.15), 10x (UMPlanFI, NA 0.30), 20x (UMPlanFI, NA 0.46). The samples were-temperature controlled by a Peltier-driven hot stage (Linkam, PE120). The effective temperature within the LC cell was determined by a calibration run.

Microspectroscopy. Spectroscopic absorption measurements were carried out on the BX61 with a 20x magnifying objective and the in-built halogen lamp $(100 \mathrm{~W})$. The signal was collected through a microspectroscopy port with a $200 \mu$ mfibre and an Ocean Optics QE 65000 spectrometer. This resulted in a collection spot size of around $25 \mu \mathrm{m}$.

Nuclear magnetic resonance (NMR) spectroscopy. NMR experiments were carried out at $297.2 \mathrm{~K}$ on a Bruker Avance III 400 spectrometer at frequencies of $400.13 \mathrm{MHz}$ for ${ }^{1} \mathrm{H}$ nuclei and $100.62 \mathrm{MHz}$ for ${ }^{13} \mathrm{C}$ nuclei or on a Bruker Avance 400 spectrometer with a BBIz $5 \mathrm{~mm}$ probe at a frequency of $400.13 \mathrm{MHz}$ for ${ }^{1} \mathrm{H} \mathrm{nu}-$ clei. Spectra were calibrated to the residual solvent peak of $\mathrm{CDCl}_{3}$ (7.26 ppm ${ }^{1} \mathrm{H}$ NMR; $\left.77.16 \mathrm{ppm}{ }^{13} \mathrm{C} \mathrm{NMR}\right) .{ }^{75}$

Transmission electron microscopy (TEM). TEM was carried out on a Philips/FEI CM12 with a LaB 6 source that was operated at $120 \mathrm{kV}$ accelerating voltage. Size analysis was carried out using imageJ.
Free energy modeling with coupled Allen-Cahn and CahnHilliard equations The relevant equations are given by: ${ }^{76}$

$$
\begin{aligned}
& \frac{\partial c}{\partial t}=\nabla \cdot D_{c} \nabla\left(\frac{\partial f(c, \eta)}{\partial c}-\epsilon_{c} \nabla^{2} c\right) \\
& \frac{\partial \eta}{\partial t}=D_{\eta}\left(\epsilon_{\eta} \nabla^{2} \eta-\frac{\partial f(c, \eta)}{\partial \eta}\right)
\end{aligned}
$$

where the composition $(c)$ and order $(\eta)$ are understood to be timedependent fields: $c(\vec{x}, t), \eta(\vec{x}, t) . D_{c}$ and $D_{\eta}$ set the time scales for the diffusion of NP and the rate of ordering of the mesogens and are assumed to be constant in the simulations. Interfaces separating two regions of differing compositions are associated with large $|\nabla c|$ and the width of that interface scales with $\sqrt{\epsilon_{c} / f_{\max }}\left(\epsilon_{c}\right.$ is the square-gradient coefficient) where $f_{\max }$ is maximum value of the free energy $f(c, \eta)$ in the interfacial region. The interfacial tension scales as $\sqrt{\epsilon_{c} f_{\max }}$. Scaling for the order-disorder interface is the same but with subscripts changed. In the simulation, an initial uniform composition and order were chosen as initial conditions to which a small amount of noise was added. The two equations 1 were then updated in a leap-frog process: one field is updated with its own value at $-\Delta t$ and the other's value at $-\Delta t / 2$. A semi-implicit spectral method was employed. ${ }^{77}$

\section{References}

1. Lagerwall, J. P. F. \& Scalia, G. A new era for liquid crystal research: Applications of liquid crystals in soft matter nano, bio- and microtechnology. Curr. Appl. Phys. 12, 1387-1412 (2012).

2. Wang, L. \& Li, Q. Stimuli-Directing Self-Organized 3D LiquidCrystalline Nanostructures: From Materials Design to Photonic Applications. Adv. Funct. Mater. 26, 10-28 (2016).

3. Kato, T., Uchida, J., Ichikawa, T. \& Sakamoto, T. Functional Liquid Crystals towards the Next Generation of Materials. Angewandte Chemie-International Edition 57, 4355-4371 (2018).

4. Blanc, C., Coursault, D. \& Lacaze, E. Ordering nano- and microparticles assemblies with liquid crystals. Liq. Cryst. Rev. 1, 83-109 (2013).

5. Smalyukh, I. I. Liquid Crystal Colloids. Annu. Rev. Condens. Matter Phys. 9, 207-226 (2018).

6. Dierking, I. From colloids in liquid crystals to colloidal liquid crystals 46, 2057-2074 (2019)

7. Tkalec, U. \& Musevic, I. Topology of nematic liquid crystal colloids confined to two dimensions. Soft Matter 9, 8140-8150 (2013).

8. Poulin, P., Stark, H., Lubensky, T. \& Weitz, D. Novel colloidal interactions in anisotropic fluids. Science 275, 1770-1773 (1997).

9. Musevic, I., Skarabot, M., Tkalec, U., Ravnik, M. \& Zumer, S. Two-dimensional nematic colloidal crystals self-assembled by topological defects. Science 313, 954-958 (2006).

10. Lapointe, C. P., Mason, T. G. \& Smalyukh, I. I. ShapeControlled Colloidal Interactions in Nematic Liquid Crystals. Science 326, 1083-1086 (2009).

11. Mundoor, H., Senyuk, B. \& Smalyukh, I. I. Triclinic nematic colloidal crystals from competing elastic and electrostatic interactions. Science 352, 69-73 (2016).

12. van der Asdonk, P. \& Kouwer, P. H. J. Liquid crystal templating as an approach to spatially and temporally organise soft matter. Chem. Soc. Rev. 46, 5935-5949 (2017).

13. Wang, X., Miller, D. S., Bukusoglu, E., de Pablo, J. J. \& Abbott, N. L. Topological defects in liquid crystals as templates for molecular self-assembly. Nat. Mater. 15, 106+ (2016).

14. Skarabot, M. \& Musevic, I. Direct observation of interaction of nanoparticles in a nematic liquid crystal. Soft Matter 6, 54765481 (2010). 
15. Tomar, V., Roberts, T. F., Abbott, N. L., Hernandez-Ortiz, J. P. \& de Pablo, J. J. Liquid Crystal Mediated Interactions Between Nanoparticles in a Nematic Phase. Langmuir 28, 6124-6131 (2012).

16. Ryzhkova, A. V. \& Musevic, I. Particle size effects on nanocolloidal interactions in nematic liquid crystals. Phys. Rev. E 87 (2013).

17. Draper, M. et al. Self-assembly and shape morphology of liquidcrystalline gold metamaterials. Adv. Funct. Mater. 21, 12601278 (2011).

18. Podoliak, N., Bartczak, D., Buchnev, O., Kanaras, A. G. \& Kaczmarek, M. High optical non linearity of nematic liquid crystals doped with gold nanoparticles. J. Phys. Chem. C 116, 12934-12939 (2012).

19. Dintinger, J. et al. A Self-Organized Anisotropic Liquid-Crystal Plasmonic Metamaterial. Adv. Mater. 25, 1999-2004 (2013).

20. Choudhary, A., Singh, G. \& Biradar, A. M. Advances in gold nanoparticle-liquid crystal composites. Nanoscale 6, 7743-7756 (2014).

21. Quint, M. T. et al. All-optical switching of nematic liquid crystal films driven by localized surface plasmons. Opt. Express 23, 6888-6895 (2015).

22. Zhang, Y., Liu, Q., Mundoor, H., Yuan, Y. \& Smalyukh, I. I. Metal Nanoparticle Dispersion, Alignment, and Assembly in Nematic Liquid Crystals for Applications in Switchable Plasmonic Color Filters and E-Polarizers. ACS Nano 9, 3097-3108 (2015).

23. Atorf, B. et al. Liquid Crystals and Precious Metal: from Nanoparticle Dispersions to Functional Plasmonic Nanostructures. Liq. Cryst. 44, 1929-1947 (2017).

24. Gabinet, U. R. \& Osuji, C. O. Optical materials and metamaterials from nanostructured soft matter. Nano Res. 12, 2172-2183 (2019).

25. Stratford, K., Henrich, O., Lintuvuori, J. S., Cates, M. E. \& Marenduzzo, D. Self-assembly of colloid-cholesteric composites provides a possible route to switchable optical materials. Nat. Commun. 5, 3954 (2014).

26. Lewandowski, W., Lojewska, T., Szustakiewicz, P., Mieczkowski, J. \& Pociecha, D. Reversible switching of structural and plasmonic properties of liquid-crystalline gold nanoparticle assemblies. Nanoscale 8, 2656-2663 (2016).

27. Baginski, M., Szmurlo, A., Andruszkiewicz, A., Wojcik, M. \& Lewandowski, W. Dynamic self-assembly of nanoparticles using thermotropic liquid crystals. Liq. Cryst. 43, 2391-2409 (2016).

28. Mitov, M., Portet, C., Bourgerette, C., Snoeck, E. \& Verelst, M. Long-range structuring of nanoparticles by mimicry of a cholesteric liquid crystal. Nat. Mater. 1, 229-231 (2002).

29. Zeng, X. et al. 3D Ordered Gold Strings by Coating Nanoparticles with Mesogens. Adv. Mater. 21, 1746-1750 (2009).

30. Gardner, D. F., Evans, J. S. \& Smalyukh, I. I. Towards Reconfigurable Optical Metamaterials: Colloidal Nanoparticle SelfAssembly and Self-Alignment in Liquid Crystals. Mol. Cryst. Liq. 545, 1227-1245 (2011).

31. Gharbi, M. A. et al. Reversible Nanoparticle Cubic Lattices in Blue Phase Liquid Crystals. ACS Nano 10, 3410-3415 (2016).

32. Zhang, S., Pelligra, C. I., Feng, X. \& Osuji, C. O. Directed Assembly of Hybrid Nanomaterials and Nanocomposites. Adv. Mater. 30, 1705794 (2018).

33. Araya, K. \& Iwasaki, K. Solubility parameters of liquid crystals. Mol. Cryst. Liq. Cryst. 392, 49-57 (2002).

34. Goodby, J. W., Mandle, R. J., Davis, E. J., Zhong, T. \& Cowling, S. J. What makes a liquid crystal? The effect of free volume on soft matter. Liq. Cryst. 42, 593-622 (2015).

35. Qi, H., Kinkead, B., Marx, V. M., Zhang, H. R. \& Hegmann, T. Miscibility and Alignment Effects of Mixed Monolayer Cyanobiphenyl Liquid-Crystal-Capped Gold Nanoparticles in Nematic Cyanobiphenyl Liquid Crystal Hosts. Chemphyschem 10, 1211-1218 (2009).
36. Milette, J., Toader, V., Reven, L. \& Lennox, R. B. Tuning the miscibility of gold nanoparticles dispersed in liquid crystals via the thiol-for-DMAP reaction. J. Mater. Chem. 21, 9043-9050 (2011).

37. Milette, J. et al. A Molecular and Thermodynamic View of the Assembly of Gold Nanoparticles in Nematic Liquid Crystal. Langmuir 29, 1258-1263 (2013).

38. Soule, E. R., Milette, J., Reven, L. \& Rey, A. D. Phase equilibrium and structure formation in gold nanoparticles-nematic liquid crystal composites: experiments and theory. Soft Matter 8, 2860-2866 (2012).

39. Gorkunov, M. V. et al. Phase separation effects and the nematicisotropic transition in polymer and low molecular weight liquid crystals doped with nanoparticles. Soft Matter 9, 3578-3588 (2013).

40. Meeker, S., Poon, W., Crain, J. \& Terentjev, E. Colloid-liquidcrystal composites: An unusual soft solid. Physical Review E 61, R6083-R6086 (2000).

41. Vollmer, D. et al. Formation of self-supporting reversible cellular networks in suspensions of colloids and liquid crystals. Langmuir 21, 4921-4930 (2005)

42. Bukusoglu, E., Pal, S. K., de Pablo, J. J. \& Abbott, N. L. Colloid-in-liquid crystal gels formed via spinodal decomposition. Soft Matter 10, 1602-1610 (2014).

43. Bartolo, M., Amaral, J. J., Hirst, L. S. \& Ghosh, S. Directed assembly of magnetic and semiconducting nanoparticles with tunable and synergistic functionality. Sci. Rep. 9, 1-11 (2019).

44. Sudha, D. G., Ochoa, J. \& Hirst, L. S. Colloidal aggregation in anisotropic liquid crystal solvent. Soft Matter 17, 7532-7540 (2021).

45. Milette, J. et al. Reversible long range network formation in gold nanoparticle - nematic liquid crystal composites. Soft Matter $\mathbf{8}$, 173-179 (2012).

46. Riahinasab, S. T. et al. Nanoparticle-based hollow microstructures formed by two-stage nematic nucleation and phase separation. Nat. Commun. 10, 894 (2019).

47. Rodarte, A. L., Pandolfi, R. J., Ghosh, S. \& Hirst, L. S. Quantum dot/liquid crystal composite materials: self-assembly driven by liquid crystal phase transition templating. J. Mater. Chem. C 1, 5527-5532 (2013).

48. Yang, Y., Serrano, L. A. \& Guldin, S. A versatile aunp synthetic platform for decoupled control of size and surface composition. Langmuir 34, 6820-6826 (2018). PMID: 29768005.

49. Qi, H. \& Hegmann, T. Formation of periodic stripe patterns in nematic liquid crystals doped with functionalized gold nanoparticles. J. Mater. Chem. 16, 4197-4205 (2006).

50. Petrov, P. \& Terentjev, E. Formation of cellular solid in liquid crystal colloids. Langmuir 17, 2942-2949 (2001).

51. Anderson, V., Terentjev, E., Meeker, S., Crain, J. \& Poon, W. Cellular solid behaviour of liquid crystal colloids - 1. Phase separation and morphology. Eur. Phys. J. E 4, 11-20 (2001).

52. Preibisch, S., Saalfeld, S. \& Tomancak, P. Globally optimal stitching of tiled 3D microscopic image acquisitions. Bioinformatics (Oxford, England) 25, 1463-5 (2009).

53. Shannon, C. E. A Mathematical Theory of Communication. Bell System Technical Journal 27, 379-423 (1948).

54. Mac Fhionnlaoich, N. \& Guldin, S. Information entropy as a reliable measure of nanoparticle dispersity. Chem. Mater. 32, 3701-3706 (2020).

55. Boettinger, W., Warren, J., Beckermann, C. \& Karma, A. Phase-field simulation of solidification. Annu. Rev. Mater. Res. 32, 163-194 (2002).

56. Bates, F. Polymer-Polymer Phase-Behavior. Science 251, 898905 (1991). 
57. Walheim, S., Boltau, M., Mlynek, J., Krausch, G. \& Steiner, U. Structure formation via polymer demixing in spin-cast films. Macromolecules 30, 4995-5003 (1997).

58. Nakanishi, K. Pore Structure Control of Silica Gels Based on Phase Separation. J. Porous Mater. 4, 67-112 (1997).

59. Vaynzof, Y. et al. Surface-Directed Spinodal Decomposition in Poly[3-hexylthiophene] and C-61-Butyric Acid Methyl Ester Blends. ACS Nano 5, 329-336 (2011).

60. Erlebacher, J., Aziz, M., Karma, A., Dimitrov, N. \& Sieradzki, K. Evolution of nanoporosity in dealloying. Nature 410, 450 453 (2001).

61. Geslin, P.-A., Mccue, I., Gaskey, B., Erlebacher, J. \& Karma, A. Topology-generating interfacial pattern formation during liquid metal dealloying. Nat. Commun. 6, 8887 (2015).

62. Carter, W. C. \& Johnson, W. C. The Selected Works of John W. Cahn (John Wiley \& Sons, Inc., 2013).

63. Mac Fhionnlaoich, N., Qi, R. \& Guldin, S. Application of the spatial distribution function to colloidal ordering. Langmuir $\mathbf{3 5}$ 16605-16611 (2019).

64. Lubensky, T., Pettey, D., Currier, N. \& Stark, H. Topological defects and interactions in nematic emulsions. Phys. Rev. E 57, 610-625 (1998).

65. Stark, H. Physics of colloidal dispersions in nematic liquid crystals. Phys. Rep. 351, 387-474 (2001).

66. Milette, J. et al. Reversible long-range patterning of gold nanoparticles by smectic liquid crystals. Soft Matter 8, 65936598 (2012).

67. Grzelczak, M., Vermant, J., Furst, E. M. \& Liz-Marzan, L. M. Directed Self-Assembly of Nanoparticles. ACS Nano 4, 35913605 (2010).

68. Klajn, R., Bishop, K. J. M. \& Grzybowski, B. A. Lightcontrolled self-assembly of reversible and irreversible nanoparticle suprastructures. Proc. Natl. Acad. Sci. U.S.A. 104, 1030510309 (2007).

69. Montelongo, Y., Yetisen, A. K., Butt, H. \& Yun, S.-H. Reconfigurable optical assembly of nanostructures. Nat. Commun. 7, 12002 (2016).

70. Fan, J. A. et al. Self-Assembled Plasmonic Nanoparticle Clusters. Science 328, 1135-1138 (2010).

71. Mann, S. Self-assembly and transformation of hybrid nano-objects and nanostructures under equilibrium and nonequilibrium conditions. Nat. Mater. 8, 781-792 (2009).
72. Marchetti, M. C. et al. Hydrodynamics of soft active matter. Rev. Mod. Phys. 85, 1143-1189 (2013).

73. Menzel, A. M. Tuned, driven, and active soft matter. Phys. Rep. 554, 1-45 (2015).

74. Lavrentovich, O. D. Active colloids in liquid crystals. Curr. Opin. Colloid Interface Sci. 21, 97-109 (2016).

75. Fulmer, G. R. et al. NMR Chemical Shifts of Trace Impurities: Common Laboratory Solvents, Organics, and Gases in Deuterated Solvents Relevant to the Organometallic Chemist. Organometallics 29, 2176-2179 (2010).

76. Balluffi, R., Allen, S. \& Carter, W. Kinetics of Materials (Blackwell Science Publ, 2005).

77. Chen, L. Phase-field models for microstructure evolution. Annu. Rev. Mater. Res. 32, 113-140 (2002).

\section{Acknowledgements}

NMF acknowledges funding by the EPSRC under a Doctoral Training Partnership (EP/M507970/1). YY is grateful to University College London for support through an Overseas Research Scholarship. LSG acknowledges funding from the European Union's Horizon 2020 research and innovation programme under grant agreement No 633635 (DIACHEMO). NBT is grateful for financial support from the 4TU.High-Tech Materials research programme 'New Horizons in designer materials' (www.4tu.nl/htm), and for discussions on this work with Paul van der Schoot, Andela Saric, Stefan Paquay, and Wouter Ellenbroek. SG is grateful for support by the German National Academy of Sciences Leopoldina, Fellowship LPDS2012-13 and by a start-up fund from University College London. The authors thank Prof Ullrich Steiner for valuable feedback on the manuscript.

\section{Author contributions}

NMF and SG carried out the aggregate formation experiments and analysed data. SS, LAS, and HF synthesised and characterised the ligand and contributed to NP solvation aspects. YY and MN synthesised the NPs. YY, KH, and PJS characterised the NPs. NBT carried out molecular dynamics simulations. WCC modeled the phase transition process. FS and SG designed the experiment. NMF, SS, NBT, HF, FS, and SG wrote the manuscript. All authors discussed the results and provided comments on the manuscript. Correspondence and requests for materials should be addressed to SG. 